\title{
microRNA-186 inhibits cell proliferation and induces apoptosis in human esophageal squamous cell carcinoma by targeting SKP2
}

\author{
Wei He${ }^{1}$, Jianfang Feng ${ }^{2}$, Yan Zhang ${ }^{1}$, Yuanyuan Wang ${ }^{3}$, Wenqiao Zang ${ }^{3}$ and Guogiang Zhao ${ }^{3}$
}

miR-186 has been demonstrated to have a significant role as a tumor suppressor in many types of cancers. Nevertheless, its biological function in esophageal squamous cell carcinoma (ESCC) remains unknown. In the present study, we found that the expression level of miR-186 was downregulated in ESCC in comparison with the adjacent normal tissues and was significantly associated with differentiation level, TNM stage, and lymph node metastasis of ESCC. Functional experiments revealed that enforced overexpression of miR-186 in ESCC cells suppressed the proliferation, invasion, and induced the apoptosis of cells. Luciferase reporter assay and western blotting analysis were performed to verify the target gene regulated by miR-186, SKP2. Our findings established that the miR-186 has a suppressive role in ESCC progression via SKP2-mediated pathway, and this implies that miR-186 could be a potential therapeutic target for ESCC.

Laboratory Investigation (2016) 96, 317-324; doi:10.1038/labinvest.2015.134; published online 16 November 2015

Esophageal cancer is one of the most common digestive tract malignancies in China, and esophageal squamous cell carcinoma (ESCC) is the dominant histological type. Despite improved prognosis owing to advances in diagnosis, surgery, and chemoradiotherapy, ESCC still ranks as the fourth leading cause of cancer death in China and seventh in the world because of its chemoresistance and tendency for metastasis. ${ }^{1}$ With further understanding of cancer biology, more and more researchers have focused on novel therapeutic approaches. Oligonucleotide therapies using microRNA (miRNA), small interfering RNA (siRNA), or ribozyme have attracted particular attention because of their specificity and safety. ${ }^{2-4}$

miRNAs, a class of small non-coding single-stranded RNAs that are 20-22 nucleotides long, are involved in multiple cellular biological processes, such as differentiation, proliferation, apoptosis, and migration by targeting the $3^{\prime}$ untranslated region (UTR) of mRNAs of target genes in a sequence-specific manner. ${ }^{5-7}$ It has also been shown that miRNAs have a crucial role in carcinogenesis and cancer progression. ${ }^{8}$ Previous studies have shown that miR-186 functioned as a tumor-suppressing miRNA in non-small cell lung carcinoma (NSCLC).$^{9-11}$ miR-186 could inhibit cell-cycle progression, proliferation, migration, and invasion of NSCLC cells by targeting different genes, and the downregulation of miR-186 was correlated with poorer survival of NSCLC patients. Zhao et al. ${ }^{12}$ found that ESCC patients at the same TNM stage but with different prognosis showed different miRNAs expression levels. The expression level of miR-186 in ESCC patients with good prognosis is almost 15 times as much as in those with poor prognosis. However, the mechanism of miR-186 as a tumor suppressor in the progression of ESCC remains unclear.

Cell-cycle regulation, tightly regulated by cyclin-dependent kinases (CDKs) and CDK inhibitors (CKIs), is critical for cell proliferation and carcinogenesis. ${ }^{13}$ S-phase kinase-associated protein 2 (SKP2), a part of SKP1-CUL1-F-box (SCF) complexes, is the specificity factor of an E3 ligase involved in cell-cycle regulation through degradation of the CKIs, ${ }^{14}$ such as $\mathrm{p} 21^{\mathrm{cip} 1}, \mathrm{p} 27^{\mathrm{kip} 1}$, and $\mathrm{p} 57^{\mathrm{kip} 2}$. Skp2 has a critical role in regulating many cellular processes such as cell-cycle regulation, cell proliferation, apoptosis, differentiation, and survival, all of which are closely related to cancer development through degradation of its substrates. ${ }^{14}$ As most of these substrates are tumor-suppressor proteins, Skp2 is considered to function as an oncogene.

In the present study, the relationship between miR-186 expression and clinicopathological features in ESCC was

1Department of Oncology, The First Affiliated Hospital of Zhengzhou University, Zhengzhou, China; ${ }^{2}$ Experimental Teaching Center of Medical Functional Science, Medical College of Henan University of Science and Technology, Luoyang, China and ${ }^{3}$ College of Basic Medical Sciences, Zhengzhou University, Zhengzhou, China

Correspondence: Professor W He, Department of Oncology, The First Affiliated Hospital of Zhengzhou University, No.1, Jianshe Road, Zhengzhou 450052, China or Professor G Zhao, College of Basic Medical Sciences, Zhengzhou University, Zhengzhou 450001, China.

E-mail: hewei726@zzu.edu.cn or zhaogq@zzu.edu.cn

Received 20 February 2015; revised 19 September 2015; accepted 25 September 2015 
Table 1 Expression of miR-186 and SKP2 mRNA in ESCC cases

\begin{tabular}{|c|c|c|c|c|c|}
\hline \multirow[t]{2}{*}{ Parameter } & \multirow[t]{2}{*}{$n$} & \multicolumn{2}{|l|}{ miR-186 } & \multicolumn{2}{|c|}{ SKP2 mRNA } \\
\hline & & Expression level & $P$-value & Expression level & $P$-value \\
\hline \multicolumn{6}{|l|}{ Gender } \\
\hline Male & 41 & $0.5452 \pm 0.1304$ & 0.997 & $1.9596 \pm 0.2148$ & 0.743 \\
\hline Female & 23 & $0.5451 \pm 0.2062$ & & $1.9775 \pm 0.1969$ & \\
\hline \multicolumn{6}{|l|}{ Age (years) } \\
\hline$<60$ & 24 & $0.5450 \pm 0.1816$ & 0.995 & $1.9658 \pm 0.2407$ & 0.996 \\
\hline$\geq 60$ & 40 & $0.5453 \pm 0.1482$ & & $1.9661 \pm 0.1875$ & \\
\hline \multicolumn{6}{|l|}{ Location } \\
\hline Low & 14 & $0.6279 \pm 0.2062$ & $0.028^{\mathrm{a}}$ & $1.8259 \pm 0.2556$ & $0.030^{\mathrm{a}}$ \\
\hline Middle & 50 & $0.5220 \pm 0.1384$ & & $2.0052 \pm 0.1751$ & \\
\hline \multicolumn{6}{|c|}{ Differentiation } \\
\hline Well & 17 & $0.6546 \pm 0.1645$ & $0.013^{\mathrm{a}}$ & $1.8089 \pm 0.2223$ & $0.025^{\mathrm{a}}$ \\
\hline Moderate & 34 & $0.5702 \pm 0.0967$ & & $1.9938 \pm 0.1859$ & \\
\hline Poor & 13 & $0.3366 \pm 0.0828$ & & $2.0988 \pm 0.0898$ & \\
\hline \multicolumn{6}{|l|}{ TNM stage } \\
\hline । & 19 & $0.6104 \pm 0.1446$ & $0.015^{\mathrm{a}}$ & $1.9278 \pm 0.2697$ & $0.031^{\mathrm{a}}$ \\
\hline$\|$ & 30 & $0.5963 \pm 0.1270$ & & $1.9294 \pm 0.1838$ & \\
\hline III & 15 & $0.3603 \pm 0.0838$ & & $2.0875 \pm 0.0953$ & \\
\hline \multicolumn{6}{|c|}{ Lymph node metastasis } \\
\hline Negative & 44 & $0.6150 \pm 0.1241$ & $<0.001^{\mathrm{a}}$ & $1.9005 \pm 0.2094$ & $<0.001^{\mathrm{a}}$ \\
\hline Positive & 20 & $0.3916 \pm 0.1185$ & & $2.1101 \pm 0.1 .062$ & \\
\hline
\end{tabular}

andicates statistical significance $(P<0.05)$.

explored in 64 pairs of ESCC and their corresponding adjacent normal tissue samples. Our findings showed that miR-186 was downregulated in ESCC tissues and was significantly associated with differentiation level, TNM stage, and lymph node metastasis of ESCC. Functional experiments revealed that overexpression of miR-186 suppressed the proliferation, invasion, and induced the apoptosis of ESCC cells through posttranscriptional downregulating SKP2 expression by targeting $3^{\prime}$ UTR of its mRNA. Our data suggested that miR-186 might have an essential role via SKP2-mediated pathway during ESCC progression.

\section{MATERIALS AND METHODS}

\section{Patient Samples}

A series of 64 surgically resected fresh ESCC and their corresponding adjacent normal tissue samples were collected at the First Affiliated Hospital of Zhengzhou University
(Zhengzhou, China) and snap-frozen in liquid nitrogen from July 2012 to January 2014. No patients had received any preoperative treatment. Clinical data of patients included in this study are detailed in Table 1. Samples used in this study were approved by the Committees for Ethical Review of the First Affiliated Hospital of Zhengzhou University.

\section{Cell Culture and Transfection}

Two ESCC cell lines Eca109 and Kyse30 were purchased from the Cell Bank of the Chinese Academy of Medical Science. Both cell lines were cultured in RPMI 1640 medium (HyClone, USA) supplemented with 10\% fetal bovine serum (HyClone) and 1\% penicillin/streptomycin (Invitrogen, USA) at $37^{\circ} \mathrm{C}$ under $5 \% \mathrm{CO}_{2}$ and saturated moisture. Het-1a, a SV40 large T-antigen-transfected normal esophageal epithelial cell line was purchased from ATCC (USA) and cultured in BEGM BulletKit Medium (Lonza, USA) at $37^{\circ} \mathrm{C}$ under $5 \%$ $\mathrm{CO}_{2}$ and saturated moisture. The sample cells were collected and counted by trypan blue exclusion using a hemocytometer. miR-186 mimics or scramble (GenePharma, China) was transfected transiently into ESCC cell lines (Eca109 and Kyse30) using Lipofectamine 2000 (Invitrogen) according to the manufacturer's instructions. The transfected amount of miR-186 mimics and scramble was 10 pmol per $1 \times 10^{3}$ cells.

\section{Real-Time PCR Analysis}

For quantitative detection of SKP2, total RNA was isolated using Trizol (Invitrogen) according to the manufacturer's instructions. cDNA was synthesized using the PrimeScript RT Reagent Kit (Takara, China). Quantitative real-time PCR analysis was performed using the Premix Ex Taq (Probe qPCR, Takara) and ROX Plus Kit (Takara) following the manufacturer's instructions. The ratio of SKP2 to $\beta$-actin was used as the normalized relative level of SKP2 expression. The primers and probe used for SKP2 cDNA detection were $5^{\prime}$-CC TTTCTGGGTGTTCTGGAT-3' (sense), 5'-CAGCCACCTGT ACATGCTTT-3' (antisense), and 5'Fam- TGCCCTGCAGAC TTTGCTAAGCA-Tamra 3' (probe). The primers and probe used for $\beta$-actin cDNA detection were 5'-CACTCTTCCA GCCTTCCTTC-3' (sense), 5'-GGATGTCCACGTCACACTT C-3' (antisense), and 5'Fam-TGCCACAGGACTCCATGCCC -Tamra 3' (probe).

For quantitative detection of miR-186, qRT-PCR analysis was performed using the Two Step Stemaim-it miR-186 qRT-PCR Quantitation Kit (Novland, China). We quantified U6 small nuclear RNA (U6 snRNA) as an endogenous control to normalize miRNA level. Relative expression level was analyzed using the $2^{-\Delta \Delta C t}$. Each sample was analyzed in triplicate on the ABI 7500 Fast thermocycler.

\section{Immunohistochemistry}

Immunohistochemical analysis for SKP2 was performed on $4-\mu \mathrm{m}$ sections. The Envision Plus detection system (Dako, USA) was used for the detection of immunostaining. The sections were deparaffinized in xylene and then microwaved 
in $10 \mathrm{mM}$ citrate buffer ( $\mathrm{pH}$ 6.0) to unmask the epitopes. Endogenous peroxidase activity was blocked by incubation with $0.03 \%$ hydrogen peroxide in methanol for $5 \mathrm{~min}$. Sections were incubated with the rabbit polyclonal anti-human SKP2 antibody $(1: 200)$ at $4{ }^{\circ} \mathrm{C}$ overnight. After gently rinsing three times with washing buffer, the sections were incubated with the peroxidase-labeled polymer conjugated to bovine anti-rabbit IgG for $30 \mathrm{~min}$ before staining for $5 \mathrm{~min}$ with 3'3-diaminobenzidine tetrahydrochloride, counterstained by hematoxylin, dehydrated, and mounted in Diatex. All the antibodies were purchased from Santa Cruz (USA).

\section{Western Blotting Analysis}

Twenty-five milligrams of protein samples were separated on a $10 \%$ SDS acrylamide gel (Bio-Rad, USA) for $1 \mathrm{~h}$ at $150 \mathrm{~V}$, and the proteins were transferred to a nitrocellulose membrane (Whatman, UK). After blocking in 5\% fat-free milk, the membrane was treated with the diluted primary antibody $(1: 800)$ at $4{ }^{\circ} \mathrm{C}$ overnight and then with the diluted secondary antibody (1:3000) for $1 \mathrm{~h}$ at room temperature. The antibody used for immunohistochemistry was subjected to western blotting. The stained membranes were visualized by enhanced chemiluminescence reaction using the ECL Plus (GE Healthcare, USA). Western blotting assay was repeated at least three times on every sample with similar results.

\section{Cell Proliferation Assay}

The effect of miR-186 on the proliferation of ESCC cells was evaluated by the MTT assay. Eca109 and KYSE30 cells were plated in 96 -well culture plates $\left(3 \times 10^{3}\right.$ per well). After $24 \mathrm{~h}$ of incubation, the cells were transfected with $30 \mathrm{pmol}$ of miR-186 mimics or scramble for 24,48 , and $72 \mathrm{~h}$. The MTT reagent $(0.5 \mathrm{mg} / \mathrm{ml}$; Sigma-Aldrich, USA) was then added to each well $(20 \mu \mathrm{l} /$ well $)$. After $4 \mathrm{~h}$ of incubation, the MTT solution was discarded, and $200 \mathrm{ml}$ of DMSO (Sigma, USA) was added. The absorbance was measured using an ELISA reader at a wavelength of $490 \mathrm{~nm}$. Each experiment was performed in triplicate.

\section{Matrigel Invasion Assay}

For invasion assay, the transfected Eca109 and KYSE30 cells were starved with serum-free medium for $24 \mathrm{~h}$ before the assay. A total of $5 \times 10^{4}$ cells were suspended in $0.5 \mathrm{ml}$ serumfree medium and loaded into the upper compartment of invasion chamber coated with Matrigel (BD Biosciences, USA). The lower compartment was filled with complete medium as chemoattractant. After $24 \mathrm{~h}$ incubation at $37^{\circ} \mathrm{C}$ in $5 \% \mathrm{CO}_{2}$, invasive cells were fixed, stained, and counted under a microscope. Triplicate independent experiments were carried out.

\section{Apoptosis Assay}

Apoptosis was assayed using the Annexin V-FITC Apoptosis Kit (Keygen, China) and Caspase 3/7 Activity Apoptosis Assay Kit (AAT Bioquest) according to the manufacturer's instructions. Briefly, the transfected Eca109 and KYSE30 cells were harvested and washed twice with PBS, followed by resuspension in Annexin-V-binding buffer. Then FITC-conjugated Annexin V and PI were added. After incubating for $10 \mathrm{~min}$ at room temperature in the dark, another binding buffer was added, and the samples were immediately analyzed using FACS Calibur flow cytometer (Beckman Coulter). For the caspase 3/7 activity apoptosis assay, cells were prepared and incubated with caspase $3 / 7$ assay solution at room temperature for $1 \mathrm{~h}$. Fluorescence intensity was monitored at $\mathrm{Ex} / \mathrm{Em}=490 / 525 \mathrm{~nm}$

\section{Construction of 3'UTR Reporter Plasmid and Luciferase Assay}

The 3'UTR of SKP2, which contains a putative target region for miR-186, was PCR amplified from genomic DNA. The SKP2 3'UTR mutant construct was generated by overlap extension PCR. Fragments were inserted between the XbaI and Xhol sites in the pmirGLO (Promega, USA).

Co-transfection of the reporter vector (pmirGLO-wt-SKP2 or pmirGLO-mut-SKP2) and miRNA (miR-186 mimics or scramble) was performed using Lipofectamine 2000 (Invitrogen). Twenty-four hours after transfection, firefly and Renilla luciferase activities were measured using the Dual Luciferase Reporter Assay Kit (Promega) according to the manufacturer's protocol.

\section{Silencing the Expression of SPK2 by siRNA}

To investigate the function of SKP2 in ESCC, the Eca109 cell line was transiently transfected with SKP2 siRNA and scramble (Qiagen, Germany) using Lipofectamine 2000 (Invitrogen) according to the manufacturer's directions. After $48 \mathrm{~h}$, the cells were analyzed to determine the effect of SKP2 siRNA on cell proliferation, invasion, and apoptosis. The silencing efficiency was confirmed by western blotting.

\section{Statistical Analysis}

Results were expressed as mean values \pm s.d. Statistical analysis was performed by Student's $t$-test, or one-way ANOVA was conducted for normally distributed data. A level of $P<0.05$ was considered to be significant.

\section{RESULTS}

The Expression of miR-186 Was Downregulated in ESCC Tissue

For this study, we measured the expression level of miR-186 in a series of 64 surgically removed fresh ESCC and their corresponding adjacent normal tissues by qRT-PCR Comparing with normal tissues, the expression level of miR-186 in ESCC tissues was downregulated by $46 \%$ $(P<0.01$; Figure 1a). Similarly, the expression of miR-186 in ESCC cell lines (Eca109 and Kyse30) was significantly lower than in normal esophageal epithelial cell line (Het-1a) $(P<0.01$; Figure $1 b)$. In addition, miR-186 expression was associated with some clinicopathological features of ESCC. As 

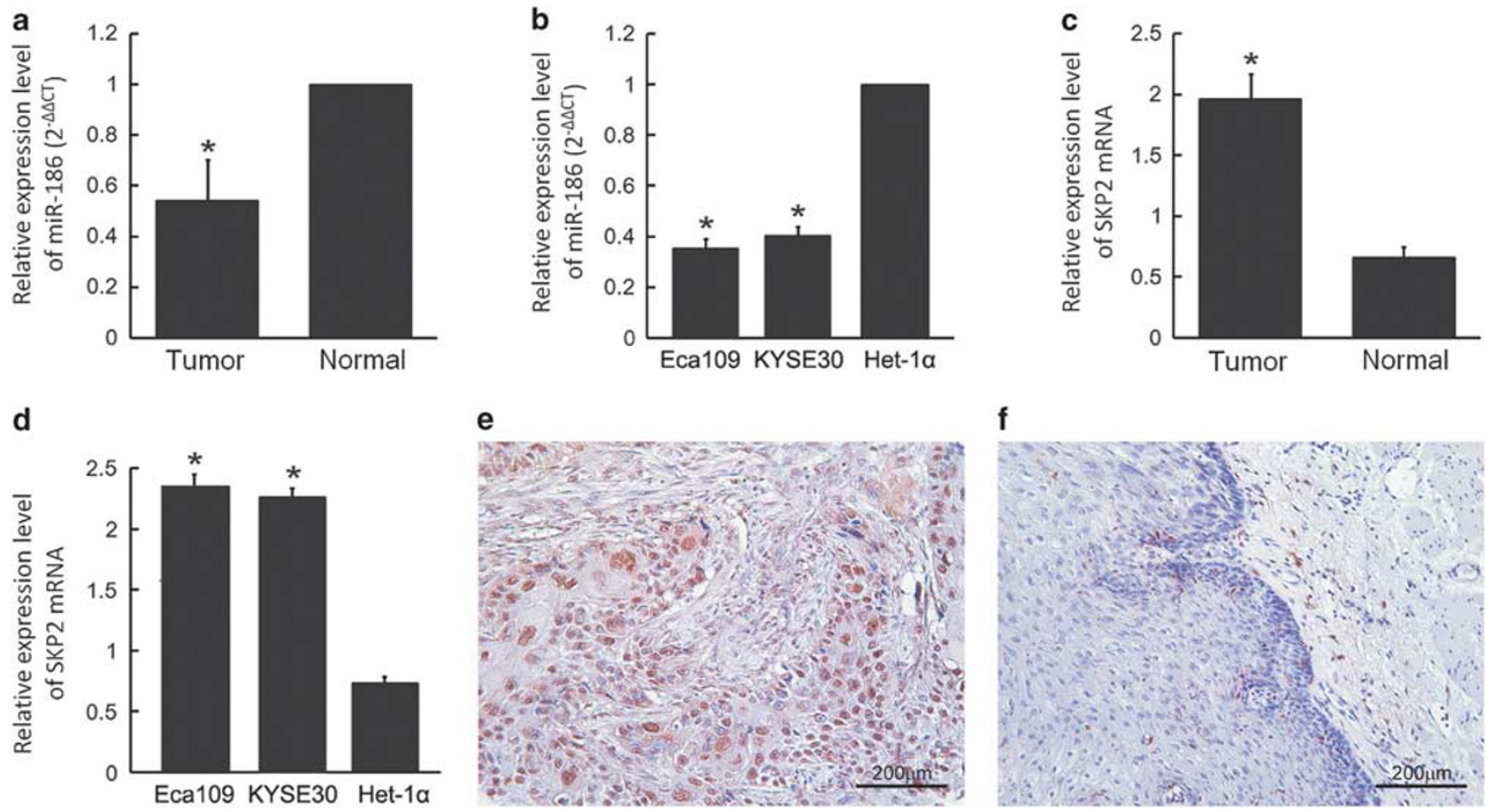

Figure 1 Expression of miR-186 and SKP2 in ESCC. (a) The relative expression of miR-186 (normalized to U6) was detected in 64 ESCC tissues and matched adjacent normal tissues. (b) The relative expression of miR-186 was detected in two ESCC cell lines and a normal esophageal epithelial cell line. (c) The relative expression of SKP2 mRNA (normalized to $\beta$-actin) was detected in 64 ESCC tissues and matched adjacent normal tissues. (d) The relative expression of SKP2 was detected in two ESCC cell lines and a normal esophageal epithelial cell line. (e) Immunohistochemistry of SKP2 protein in ESCC tissue. (f) Immunohistochemistry of SKP2 protein in normal esophagus tissue. ${ }^{*} P<0.05$.

shown in Table 1, the expression level of miR-186 in ESCC was inversely correlated with differentiation grade $(P=0.013)$, TNM stage $(P=0.015)$, and lymph node metastasis $(P<0.001)$ significantly but not associated with either age or gender.

\section{The Expression of SKP2 Was Upregulated in ESCC Tissue} In qRT-PCR analysis, the expression of SKP2 mRNA in ESCC tissues was higher than in adjacent non-tumor tissues $(P<0.01$; Figure 1c). Also, similar results were observed in ESCC cell lines and normal esophageal epithelial cell line $(P<0.01$; Figure 1d). In addition, SKP2 expression level in ESCC tissues was correlated with differentiation grade $(P=0.025)$, TNM stage $(P=0.031)$, and lymph node metastasis $(P<0.001)$ significantly (Table 1$)$. Immunohistochemically, SKP2-positive immunostaining was observed in ESCC nuclei (Figure 1e). No staining was observed in adjacent non-tumor tissues (Figure 1f).

\section{miR-186 Suppressed Proliferation and Invasion of ESCC Cells}

To investigate the function of miR-186 in ESCC cells, Eca109 and KYSE30 cells were transfected with miR-186 mimics or scramble. As shown in Figure 2a, the miR-186 mimics increased the expression of miR-186 by 9.45 -fold in Eca109 and 8.57-fold in KYSE30 cells. MTT assay was performed to examine the effect of miR-186 on cell growth in vitro. Our data showed that the proliferation of ESCC cells was significantly suppressed by miR-186 mimics (Figure 2b). As the matrigel invasion assay shown in Figure $2 c$, miR-186 mimics significantly decreased the number of ESCC cells invaded through the matrigel. Quantitative analysis of cell numbers revealed that, in the scramble negative control (NC) and blank control groups, cells invaded through the matrigel were almost four times higher than in the miR-186 mimics group.

\section{miR-186 Induced Apoptosis of ESCC Cells}

Flow cytometry showed that the proportion of apoptotic Eca109 cells in miR-186 mimics transfection group was significantly higher than that in the scramble and blank control groups (Figure 3a, 17.4\% vs $6.7 \%$ vs 6.2\%). In KYSE30 cells, we also obtained similar result ( $14.8 \%$ vs $3.9 \%$ vs 3.4\%). Caspase 3/7 activity apoptosis assay also showed similar results. The caspase 3/7 activity of miR-186 mimics transfected cells was almost 2.5-fold as much as the control groups (Figure 3b).

\section{miR-186 Directly Targeted and Suppressed SKP2 mRNA Expression}

To explore the potential targets of miR-186, a large number of target genes were predicted by three computational 
algorithms, namely, TargetScan, miRanda, and PITA. According to previous reports, TWIST $1,{ }^{15}$ GLUT $1,{ }^{16}$ PTTG $1,{ }^{10}$ and SKP2 (Figure 4a) might be potential targets of miR-186 in ESCC. Our data showed that TWIST1 and GLUT1 mRNA expression have no significant difference between ESCC tissues and the corresponding adjacent normal tissues
(Supplementary Figures S1a and b). Meanwhile, the expression level of PTTG1 and SKP2 in ESCC tissues is significantly higher than in adjacent normal tissues (Supplementary Figure S1c, Figure 1c). We then tested whether miR-186 can influence endogenous PTTG1 or SKP2 expression. In Eca109 and KYSE30 cells, miR-186 mimics could induce a a

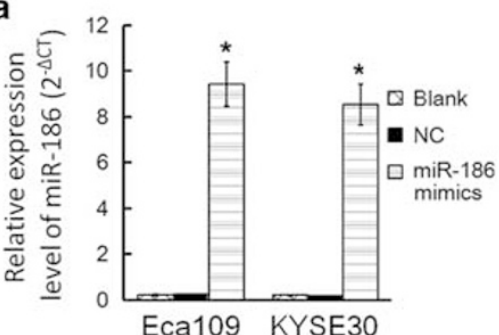

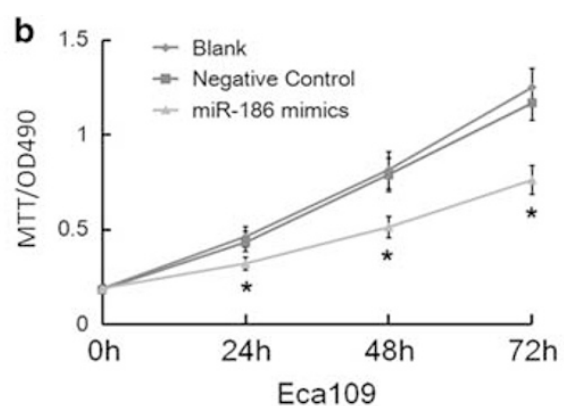

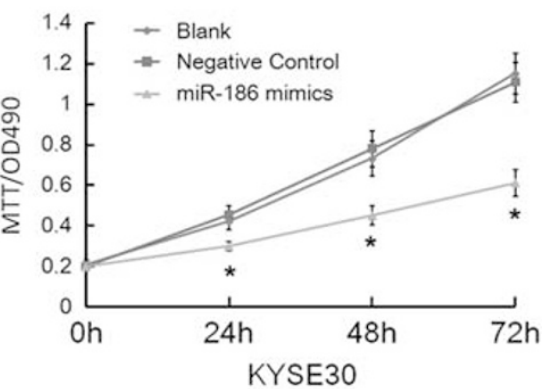

KYSE30

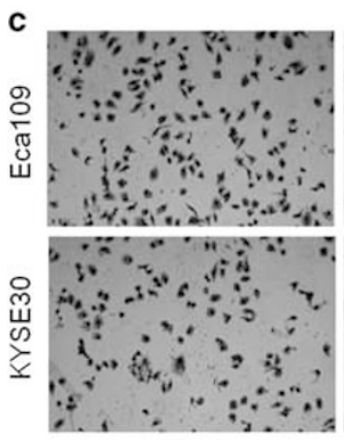

Blank

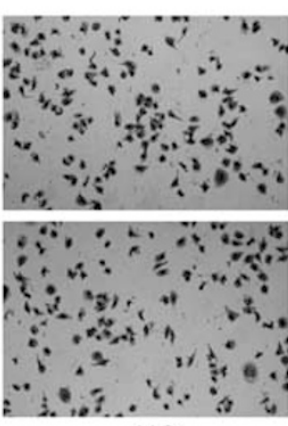

NC

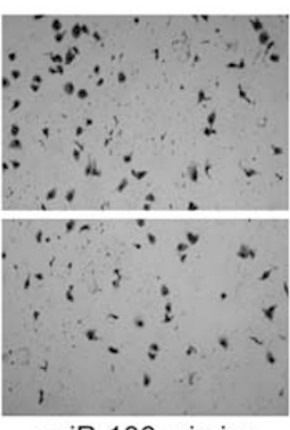

miR-186 mimics

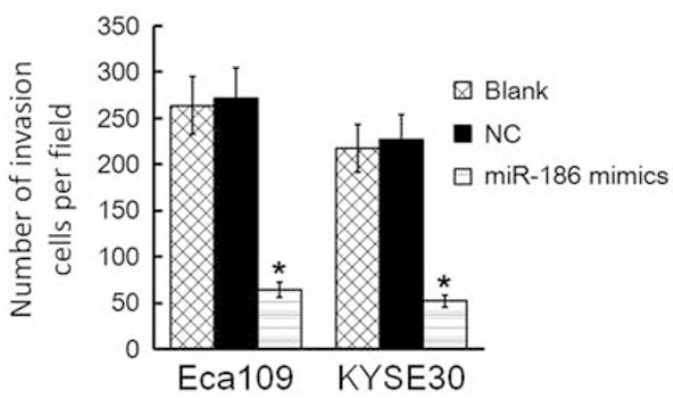

Figure 2 Upregulation of miR-186 suppressed proliferation and invasion of ESCC cells. (a) Real-time PCR analysis of miR-186 expression in Eca109 and KYSE30 cells after transfection with miR-186 mimics or scramble negative control (NC). (b) The proliferation ability of Eca109 and KYSE30 cells transfected with miR-186 mimics or NC measured by MTT assay. (c) The invasion ability of Eca109 and KYSE30 cells transfected with miR-186 mimics or NC measured by matrigel invasion assay. ${ }^{*} P<0.05$.
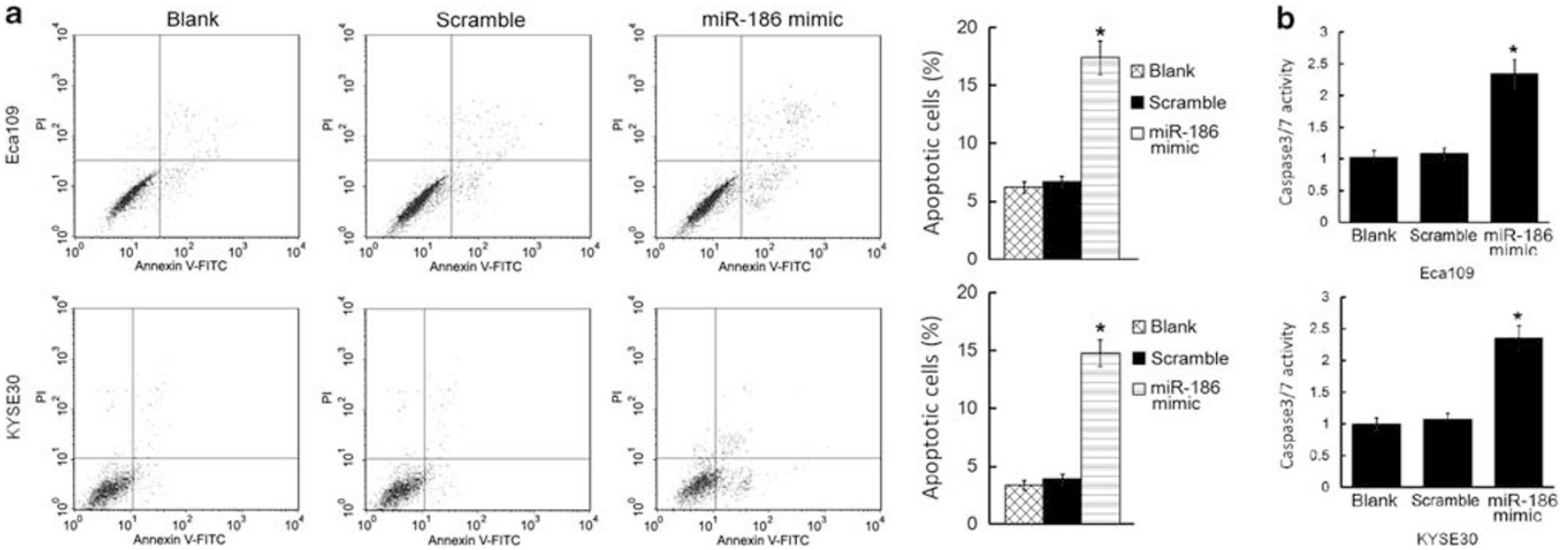

Figure 3 miR-186-induced apoptosis of ESCC cells. (a) Annexin V-FITC apoptosis assay showed that miR-186 mimics could increase the apoptosis proportion of both Eca109 and KYSE30 cells. (b) miR-186 mimics could increase the caspase 3/7 activity of both Eca109 and KYSE30 cells. ${ }^{*} P<0.05$. 
a

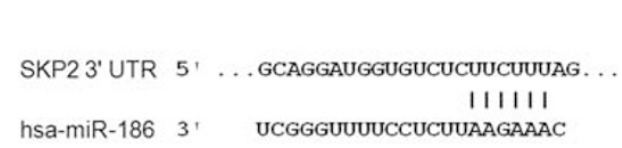

b
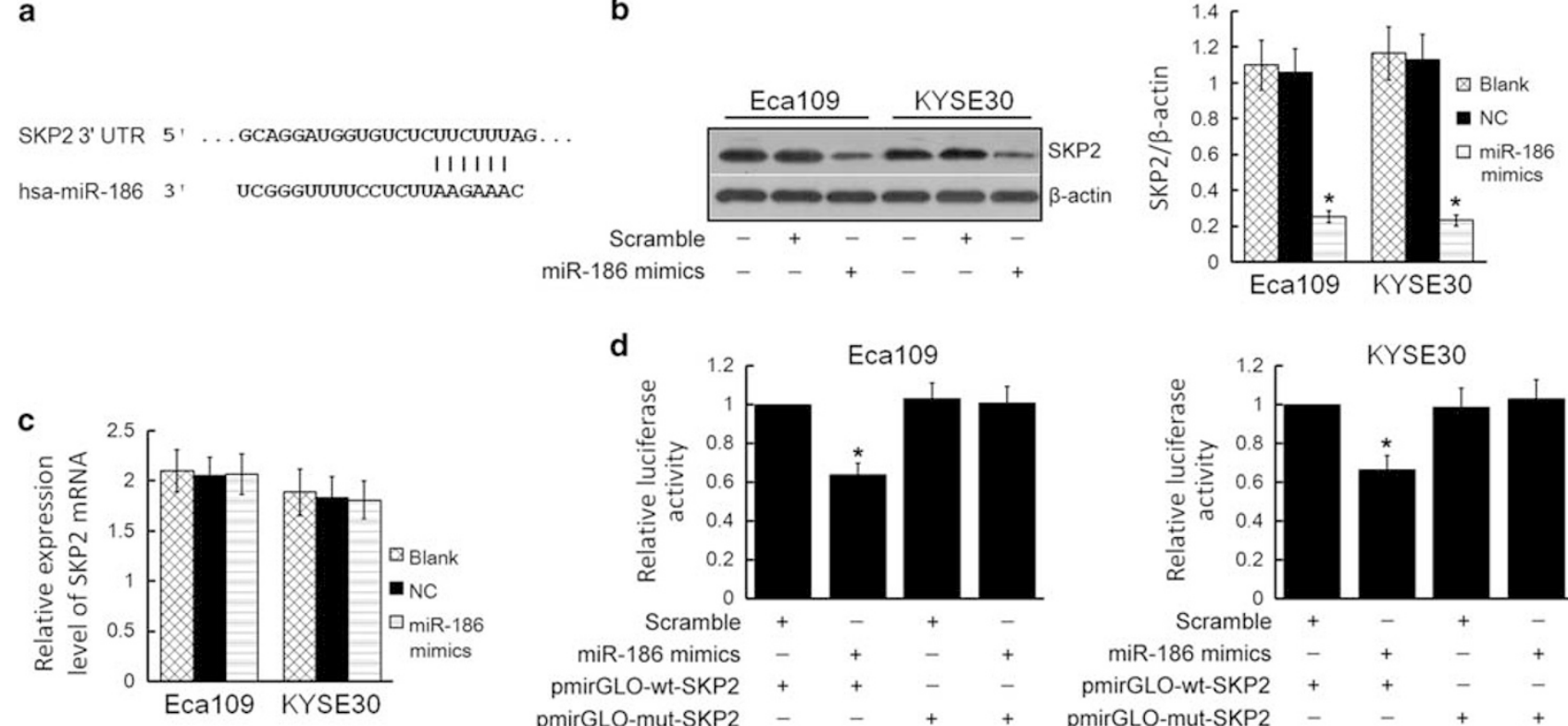

d

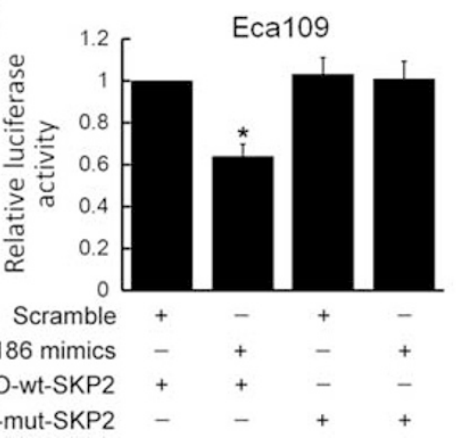

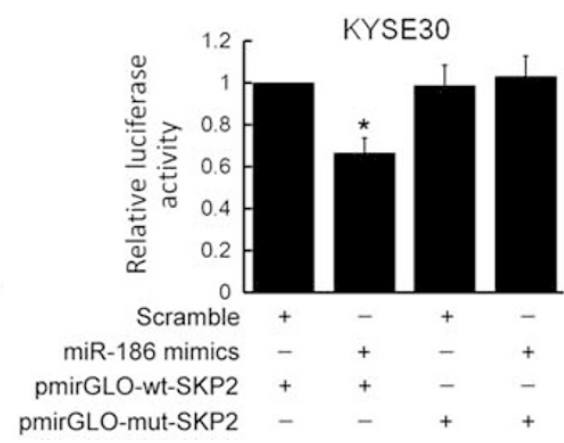

Figure 4 miR-186 targeted SKP2 to suppress its expression. (a) Computational algorithms predicted that miR-186 might target SKP2. (b) Western blotting analysis showed that miR-186 could reduce the expression of SKP2 protein. (c) Real-time PCR analysis showed that miR-186 could not affect the expression of SKP2 mRNA. (d) miR-186 mimics could significantly reduce the luciferase activity of pmirGLO-wt-SKP2 clone, compared with pmirGLO-mut-SKP2 clone. However, the scramble could not affect the luciferase activity of neither pmirGLO-wt-SKP2 nor pmirGLO-mut-SKP2 clone. ${ }^{*} P<0.05$.

reduction of endogenous SKP2 protein expression (Figure 4b) without affecting the SKP2 mRNA level (Figure 4c), but not PTTG1 (Supplementary Figure S1d). So we conclude that SKP2 could be the best target of miR-186 in ESCC. To investigate whether the effect of miR-186 on SKP2 is specific, we employed the 3'UTR luciferase reporter assay. The result showed that miR-186 mimics could significantly reduce the luciferase activity of pmirGLO-wt-SKP2 clone, compared with pmirGLO-mut-SKP2 clone. However, the scramble could not affect the luciferase activity of neither pmirGLO-wt-SKP2 nor pmirGLO-mut-SKP2 clone (Figure 4d). These results provide evidence that miR-186 can directly target the 3'UTR of SKP2 mRNA, resulting in suppression of its translation.

\section{Silencing of SKP2 Suppressed Proliferation and Invasion of ESCC Cells and Induced Apoptosis}

To investigate the function of SKP2 in ESCC cells, Eca109 cells were transfected with SKP2 siRNA. As shown in Figure 5a, the expression of SKP2 in Eca109 was suppressed by the transfection of SKP2 siRNA. The functional assays showed that, similar to miR-186, SKP2 siRNA could also suppress the proliferation, invasion, and induce the apoptosis of ESCC cells (Figures 5b-d).

\section{DISCUSSION}

To date, $>1900$ human miRNAs have been identified, which are estimated to regulate $>60 \%$ genes in our body. ${ }^{17}$ Several studies have showed that miRNAs might contribute to human carcinogenesis and cancer progression and be considered as potential novel targets for cancer diagnosis and treatment. ${ }^{2,8}$ The global miRNA expression profile in ESCC was demonstrated for the first time in $2008,{ }^{18}$ and some miRNAs have been reported to exert regulatory functions in ESCC. miRNAs, including miR-21, miR-31, miR-577, etc, act as oncogenes, ${ }^{19-21}$ while others such as Let-7, miR-98, miR-100, miR-138, miR-143, miR-150, etc, function as tumor suppressors. $^{22-27}$ However, the role of miR-186 in ESCC progression remains uninvestigated.

In the current study, we determined the miR-186 expression level in ESCC tissues and their corresponding adjacent normal tissues by real-time PCR analysis. The result showed that the expression of miR-186 is downregulated in ESCC tissues. Regarding the clinical outcome, the expression level of miR-186 in ESCC was significantly inversely correlated with differentiation grade, TNM stage, and lymph node metastasis but not associated with either age or gender, indicating that miR-186 may function as a tumor suppressor in ESCC. In addition, a series of functional experiments were conducted to verify the role miR-186 played in the progression of ESCC. Our data showed that overexpression of miR-186 could suppress the proliferation, invasion, and induce the apoptosis of ESCC cells, confirming that miR-186 acts as a tumor suppressor in the progression of ESCC.

In general, miRNAs exert their functions by binding to the $3^{\prime}$ UTR of target gene mRNA to repress its expression. ${ }^{28}$ To identify the target genes of miR-186, we explored the possible target genes of miR-186 via different computational 
a

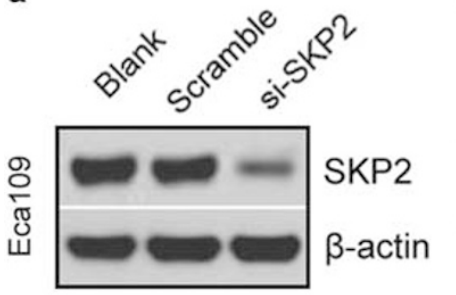

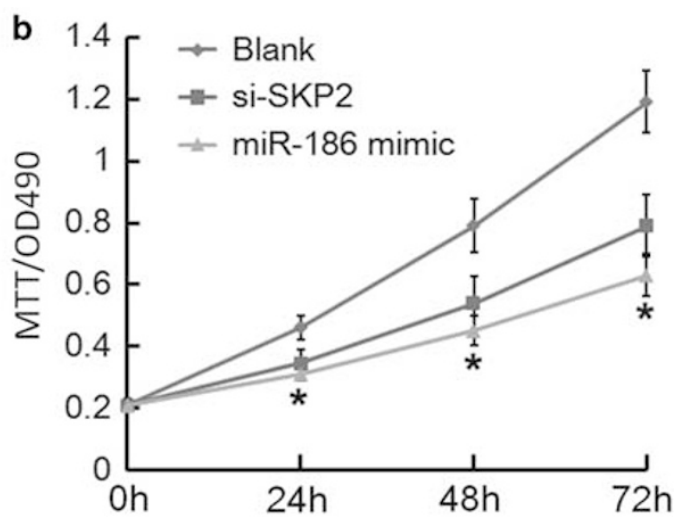

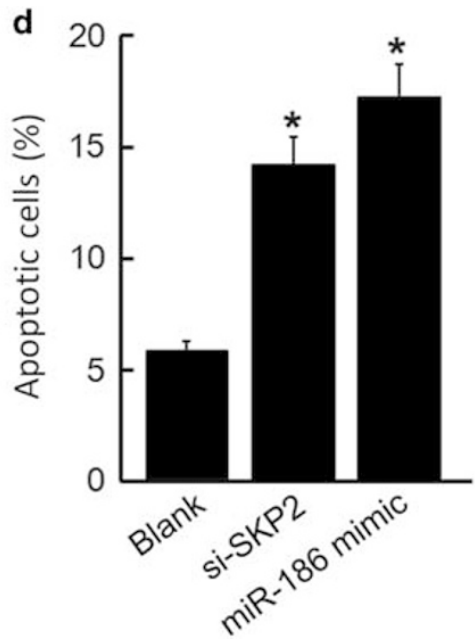

Figure 5 Downregulation of SKP2 expression suppressed proliferation and invasion of ESCC cells. (a) The expression of SKP2 in Eca109 was reduced by SKP2 siRNA. (b) The proliferation ability of Eca109 was suppressed by SKP2 siRNA. (c) The invasion ability of Eca109 was suppressed by SKP2 siRNA. (d) SKP2 siRNA could increase the apoptosis proportion of Eca109 cells. ${ }^{*} P<0.05$.

algorithms and selected SKP2, a negative regulator of the cell cycle, as our target gene of interest. In addition, the luciferase reporter assay was carried out to verify SKP2 as a target gene of miR-186. The results showed that miR-186 could remarkably decrease the luciferase intensity of the wild-type SKP2-3'UTR reporter but had no effect on the mutant one, indicating that SKP2 is a direct target gene of miR-186. Additionally, western blotting and real-time PCR analysis showed that miR-186 could significantly reduce the protein level of SKP2 in ESCC cell lines without affecting its mRNA level. Our study confirmed that miR-186 could directly target the $3^{\prime}$ UTR of SKP2 mRNA, resulting in inhibition of its translation and suppression of expression.

Several studies have revealed that SKP2 is overexpressed in various human cancers, including lymphoma, ${ }^{29}$ breast carcinoma, ${ }^{30}$ prostate cancer, ${ }^{31}$ melanoma, ${ }^{32}$ nasopharyngeal carcinoma, ${ }^{33}$ and pancreatic cancer. ${ }^{34}$ Furthermore, it has been found that the expression of SKP2 could be considered as a prognostic factor for patients with breast cancer, melanoma, and nasopharyngeal carcinoma. There are also several in vivo studies that confirm the oncogenic role of SKP2 in tumorigenesis. Xenografts of breast cancer cell lines with high SKP2 expression grow much faster than those with low expression level of SKP2. ${ }^{35}$ SKP2 knockout mice are resistant to tumor development induced by loss of tumor-suppressor genes, such as p19ARF or PTEN. ${ }^{36}$ Taken together, SKP2 has a key role in the development and progression of cancer.

A previous study showed that overexpression of SKP2 was significantly correlated with ESCC clinical stage and positive lymph node metastasis. In addition, a functional study showed that decreased expression of SKP2 in ESCC cells could inhibit the migration, invasion, colony formation of ESCC cells in vitro, and tumorigenesis in mouse models by inactivating the PI3k-Akt pathway. ${ }^{37}$ In the present study, the overexpression of SKP2 was also found in ESCC tissues. In addition, the correlation analysis showed that the expression level of SKP2 was significantly correlated with differentiation grade, TNM stage, and lymph node metastasis of ESCC. Given that SKP2 is a target gene of miR-186, we silenced the expression of SKP2 in ESCC cells directly by siRNA to determine whether miR-186 exerts its suppressing function in ESCC by inhibiting SKP2. Our results showed that SKP2 
siRNA could also suppress the proliferation, invasion, and induce the apoptosis of ESCC cells, similar to miR-186.

In conclusion, our present study demonstrated the suppressing function of miR-186 in ESCC by targeting SKP2 directly. Thus our finding warrants further investigation on the potential development of miR-186-based therapeutic approaches for ESCC.

Supplementary Information accompanies the paper on the Laboratory Investigation website (http://www.laboratoryinvestigation.org)

\section{ACKNOWLEDGMENTS}

This work was funded by the Foundation and Frontier Project of Henan Province (122300410101), Science and Technology Research Project of Henan Health Department (201303021) and Special Research Foundation of Young teachers in Zhengzhou University (1421412089).

\section{DISCLOSURE/CONFLICT OF INTEREST}

The authors declare no conflict of interest.

1. Parkin DM, Bray F, Ferlay J et al. Global cancer statistics, 2002. CA Cancer J Clin 2005;55:74-108.

2. Sibley CR, Seow Y, Wood MJ. Novel RNA-based strategies for therapeutic gene silencing. Mol Ther 2010;18:466-476.

3. Ireson CR, Kelland LR. Discovery and development of anticancer aptamers. Mol Cancer Ther 2006;5:2957-2962.

4. Guo P, Coban O, Snead NM et al. Engineering RNA for targeted siRNA delivery and medical application. Adv Drug Deliv Rev 2010;62:650-666.

5. Ambros V. The functions of animal microRNAs. Nature 2004;431: 350-355.

6. Bartel DP. microRNAs: target recognition and regulatory functions. Cell 2009;136:215-233.

7. Volinia S, Calin GA, Liu CG et al. A microRNA expression signature of human solid tumors defines cancer gene targets. Proc Natl Acad Sci USA 2006;103:2257-2261.

8. Wang $\mathrm{D}$, Qiu $\mathrm{C}$, Zhang $\mathrm{H}$ et al. Human microRNA oncogenes and tumor suppressors show significantly different biological patterns: from functions to targets. PLoS One 2010;5.

9. Cai J, Wu J, Zhang $\mathrm{H}$ et al. Mir-186 downregulation correlates with poor survival in lung adenocarcinoma, where it interferes with cell-cycle regulation. Cancer Res 2013;73:756-766.

10. Li H, Yin C, Zhang B et al. Pttg1 promotes migration and invasion of human non-small cell lung cancer cells and is modulated by Mir-186. Carcinogenesis 2013;34:2145-2155.

11. Cui G, Cui M, Li Y et al. Mir-186 targets Rock1 to suppress the growth and metastasis of NSCLC cells. Tumour Biol 2014;35:8933-8937.

12. Zhao BS, Liu SG, Wang TY et al. Screening of microRNA in patients with esophageal cancer at same tumor node metastasis stage with different prognoses. Asian Pac J Cancer Prev 2013;14:139-143.

13. Bertoli C, Skotheim JM, de Bruin RA. Control of cell cycle transcription during G1 and S phases. Nat Rev Mol Cell Biol 2013;14:518-528.

14. Frescas $\mathrm{D}$, Pagano M. Deregulated proteolysis by the F-box proteins SKP2 and beta-TrCP: tipping the scales of cancer. Nat Rev Cancer 2008;8:438-449.

15. Zhu $X$, Shen $H$, Yin $X$ et al. Mir-186 regulation of Twist1 and ovarian cancer sensitivity to cisplatin. Oncogene 2015 [e-pub ahead of print].

16. Sun P, Hu JW, Xiong WJ et al. Mir-186 regulates glycolysis through Glut1 during the formation of cancer-associated fibroblasts. Asian Pac J Cancer Prev 2014;15:4245-4250.

17. Esteller M. Non-coding RNAs in human disease. Nat Rev Genet 2011;12: 861-874.
18. Guo $Y$, Chen $Z$, Zhang $L$ et al. Distinctive microRNA profiles relating to patient survival in esophageal squamous cell carcinoma. Cancer Res 2008;68:26-33.

19. Wang N, Zhang CQ, He JH et al. Mir-21 down-regulation suppresses cell growth, invasion and induces cell apoptosis by targeting Fasl, Timp3, and Reck genes in esophageal carcinoma. Dig Dis Sci 2013;58: 1863-1870.

20. Alder $\mathrm{H}$, Taccioli $\mathrm{C}$, Chen $\mathrm{H}$ et al. Dysregulation of Mir-31 and Mir-21 induced by zinc deficiency promotes esophageal cancer. Carcinogenesis 2012;33:1736-1744.

21. Yuan $X, \mathrm{He} J$, Sun F et al. Effects and interactions of miR-577 and TSGA10 in regulating esophageal squamous cell carcinoma. Int J Clin Exp Pathol 2013;6:2651-2667.

22. Huang SD, Yuan Y, Zhuang CW et al. microRNA-98 and microRNA-214 post-transcriptionally regulate enhancer of zeste homolog 2 and inhibit migration and invasion in human esophageal squamous cell carcinoma. Mol Cancer 2012;11:51.

23. Sun J, Chen Z, Tan X et al. microRNA-99a/100 promotes apoptosis by targeting mTOR in human esophageal squamous cell carcinoma. Med Oncol 2013;30:411.

24. Gong $\mathrm{H}$, Song L, Lin C et al. Downregulation of miR-138 sustains NF-KappaB activation and promotes lipid raft formation in esophageal squamous cell carcinoma. Clin Cancer Res 2013;19: 1083-1093.

25. Ni $Y$, Meng L, Wang $L$ et al. microRNA-143 functions as a tumor suppressor in human esophageal squamous cell carcinoma. Gene 2013;517:197-204.

26. Yokobori T, Suzuki S, Tanaka $\mathrm{N}$ et al. miR-150 is associated with poor prognosis in esophageal squamous cell carcinoma via targeting the EMT inducer Zeb1. Cancer Sci 2013:104:48-54.

27. Sugimura $\mathrm{K}$, Miyata $\mathrm{H}$, Tanaka $\mathrm{K}$ et al. Let-7 expression is a significant determinant of response to chemotherapy through the regulation of IL-6/STAT3 pathway in esophageal squamous cell carcinoma. Clin Cancer Res 2012;18:5144-5153.

28. van Kouwenhove M, Kedde M, Agami R. microRNA regulation by RNAbinding proteins and its implications for cancer. Nat Rev Cancer 2011;11:644-656.

29. Seki $\mathrm{R}$, Okamura $\mathrm{T}$, Koga $\mathrm{H}$ et al. Prognostic significance of the F-box protein SKP2 expression in diffuse large b-cell lymphoma. Am J Hematol 2003;73:230-235.

30. Voduc D, Nielsen TO, Cheang MC et al. The combination of high cyclin E and SKP2 expression in breast cancer is associated with a poor prognosis and the basal phenotype. Hum Pathol 2008;39: $1431-1437$

31. Wang $\mathrm{Z}$, Gao D, Fukushima $\mathrm{H}$ et al. SKP2: a novel potential therapeutic target for prostate cancer. Biochim Biophys Acta 2012;1825:11-17.

32. Rose $A E$, Wang $G$, Hanniford $D$ et al. Clinical relevance of SKP2 alterations in metastatic melanoma. Pigment Cell Melanoma Res 2011:24:197-206.

33. Fang FM, Chien CY, Li CF et al. Effect of S-phase kinase-associated protein 2 expression on distant metastasis and survival in nasopharyngeal carcinoma patients. Int J Radiat Oncol Biol Phys 2009;73: 202-207.

34. Schuler S, Diersch S, Hamacher R et al. SKP2 confers resistance of pancreatic cancer cells towards TRAIL-induced apoptosis. Int J Oncol 2011:38:219-225.

35. Radke S, Pirkmaier A, Germain D. Differential expression of the F-box proteins SKP2 and SKP2b in breast cancer. Oncogene 2005;24: 3448-3458.

36. Lin HK, Chen Z, Wang G et al. SKP2 targeting suppresses tumorigenesis by Arf-p53-independent cellular senescence. Nature 2010;464: 374-379.

37. Wang XC, Wu YP, Ye B et al. Suppression of anoikis by SKP2 amplification and overexpression promotes metastasis of esophageal squamous cell carcinoma. Mol Cancer Res 2009;7:12-22. 